\title{
A novel cross-correlation methodology for assessing biophysical responses associated with pain
}

\author{
John Sunwoo' \\ Patjanaporn Chalacheva' \\ Maha Khaleel ${ }^{2}$ \\ Payal Shah ${ }^{2}$ \\ Richard Sposto 3 \\ Roberta M Kato ${ }^{4}$ \\ Jon Detterich ${ }^{5}$ \\ Lonnie K Zeltzer ${ }^{6}$ \\ John CWood ${ }^{1,5}$ \\ Thomas D Coates ${ }^{2}$ \\ Michael CK Khoo'
}

'Department of Biomedical Engineering, University of Southern California, Los Angeles, CA, USA; ${ }^{2}$ Hematology Section, Children's Center for Cancer, Blood

Disease and Bone Marrow Transplantation, Children's Hospital Los Angeles, Keck

School of Medicine, University of Southern California, Los Angeles, CA, USA; ${ }^{3}$ Children's Center for Cancer, Blood Disease and Bone Marrow Transplantation, Children's Hospital Los Angeles, Keck

School of Medicine, University of Southern California, Los Angeles, CA, USA; ${ }^{4}$ Division of Pediatric Pulmonology, Children's Hospital Los Angeles, Keck School of Medicine, University of Southern California, Los Angeles, CA, USA; ${ }^{5}$ Division of Pediatric Cardiology, Children's Hospital Los Angeles, Keck School of Medicine, University of Southern California, Los Angeles, CA, USA; ${ }_{6}^{6}$ Pediatric Pain and Palliative Care Program, Department of Pediatrics, Division of Hematology-Oncology, David Geffen Schoo of Medicine at the University of California Los Angeles, Los Angeles, CA, USA

Correspondence: John Sunwoo

Department of Biomedical Engineering,

University of Southern California, 1042

Downey Way, DRB I40, Los Angeles, CA

90089, USA

Tel + I 2137400827

Fax +I 2137400343

Email johnsunw@usc.edu
This article was published in the following Dove Press journal: Journal of Pain Research

Purpose: The purpose of this work was to noninvasively detect and quantify microvascular blood flow changes in response to externally applied pain in humans. The responsiveness of the microvasculature to pain stimulation might serve as an objective biomarker in diseases associated with altered pain perception and dysregulated vascular functions. The availability of such a biomarker may be useful as a tool for predicting outcome and response to treatments, particularly in diseases like sickle cell anemia where clinical manifestations are directly linked to microvascular perfusion. We, therefore, developed a method to distinguish the blood flow response due to the test stimulus from the blood flow measurement that also includes concurrent flow changes from unknown origins.

Subjects and methods: We measured the microvascular blood flow response in 24 healthy subjects in response to a train of randomly spaced and scaled heat pulses on the anterior forearm. The fingertip microvascular perfusion was measured using laser Doppler flowmetry. The cross-correlation between the heat pulses and the blood flow response was computed and tested for significance against the null distribution obtained from the baseline recording using bootstrapping method.

Results: We estimated correlation coefficients, response time, response significance, and the magnitude of vasoreactivity from microvascular blood flow responses. Based on these pain response indices, we identified strong responders and subjects who did not show significant responses.

Conclusion: The cross-correlation of a random pattern of painful stimuli with directly measured microvascular flow can detect vasoconstriction responses in a noisy blood flow signal, determine the time between stimulus and response, and quantify the magnitude of this response. This approach provided an objective measurement of vascular response to pain that may be an inherent characteristic of individual human subjects, and may also be related to the severity of vascular disorders.

Keywords: objective quantification of pain, randomized heat pulse train, laser Doppler flowmetry, cross-correlation, bootstrap test, vasoreactivity

\section{Introduction}

This study is motivated by our interest in understanding the relationship between pain, autonomic nervous system modulation, and changes in microvascular blood flow in order to develop therapeutic approaches for pain-related complications in sickle cell disease (SCD). Painful vaso-occlusive crises (VOC) are common in SCD and result in acute, as well as chronic, pain. ${ }^{1}$ Our previous studies have suggested that the autonomic nervous system (ANS) plays an important role in mediating peripheral vasoconstriction, which 
likely increases the risk of VOC. ${ }^{2-4}$ Sickle vaso-occlusion happens when local perfusion drops and red cells get trapped in the microvasculature. Pain resulting from localized VOC could trigger sympathetically mediated vasoconstriction in an expanding number of microvascular networks, producing a cascading effect that leads to large-scale VOC. ${ }^{1,4}$ Thus, microvascular perfusion is a direct biomarker of the pathology of SCD and may be at the same time an objective marker of paininduced neural activation. Thus, quantifying the association between experimental pain and local vasoconstriction using noninvasive measurements could provide us with an objective tool for predicting certain clinical outcomes in disorders where microvascular flow plays a role in the pathology itself.

A major challenge in pain research stems from the fact that pain produces sensations that are experienced differently in different individual subjects. For example, the patient may be asked "how intense is your pain, give me a number from one to ten," which is obviously subjective. We wanted to supplement this subjective response with an objectively quantifiable biomarker of the response to pain and explore peripheral vasoreactivity (VR) as a possible marker.

However, the blood flow signal contains multiple fluctuations due to various other inputs, in addition to the experimental stimulus. ${ }^{5}$ These thoughts are summarized in Figure 1 which shows a single heat pulse (the experimental stimulus) in the first row, the microvascular blood flow response measured using laser Doppler flowmetry in the second row, and the respiratory pattern in the third row. There are many periods of vasoconstriction unrelated to the pain signal that are evident in the laser Doppler tracing. One was clearly associated with a sigh (Figure 1C), some appear to be happening randomly (Figure 1B), and there is a possible response to the single painful stimulus (Figure 1A). However, it is evident from this picture that we cannot tell if a single painful stimulus is caus-

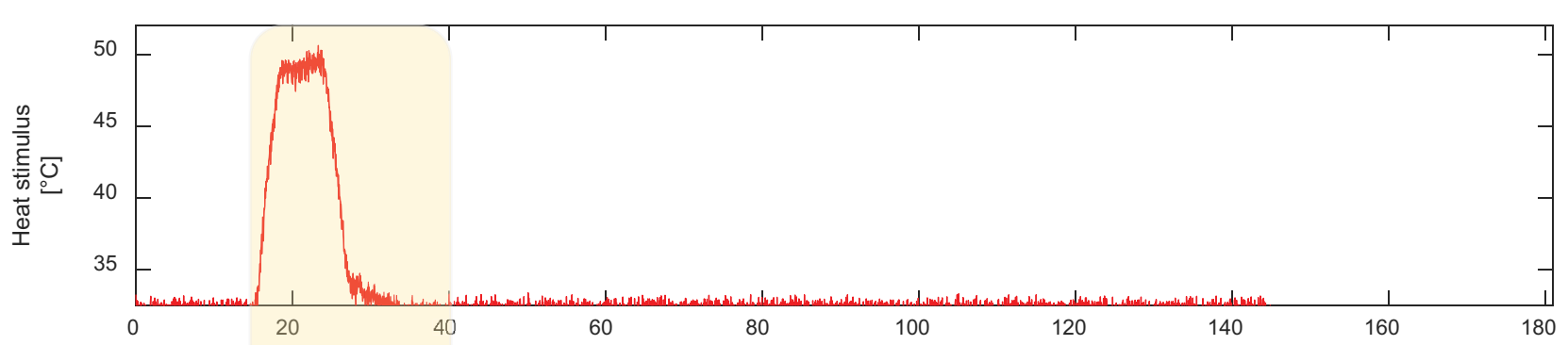

(A) Pain
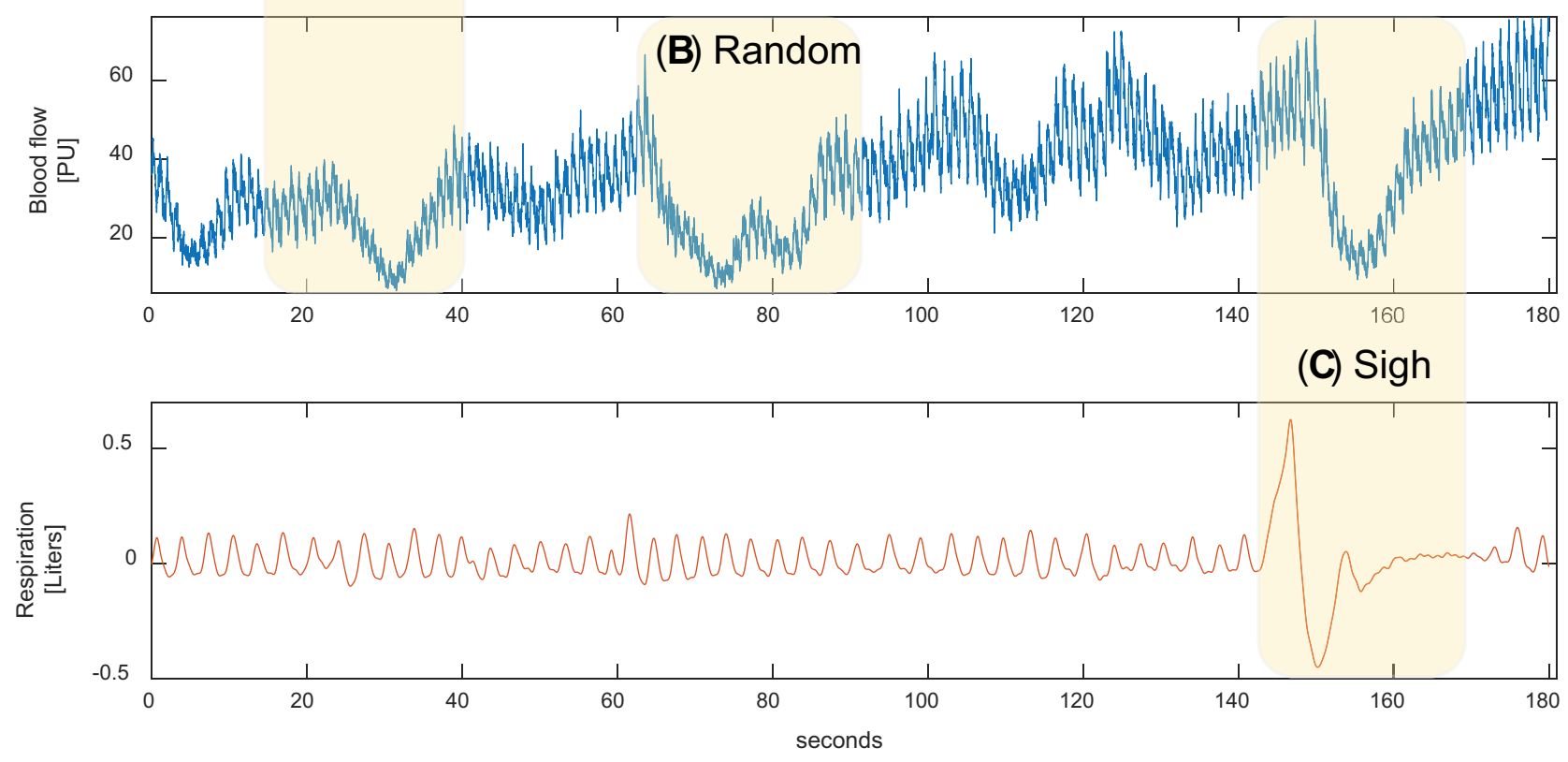

Figure I Periods of vasoconstriction due to various events.

Notes: A single heat pulse stimulus (top row) and microvascular blood flow responses (middle row), while respiratory pattern was recorded simultaneously. (A) We cannot tell if the single pain stimulus caused the vasoconstriction or whether the vasoconstriction would have occurred in the absence of stimulation, (B) because vasoconstriction can occur randomly, or (C) it can associate with a sigh event.

Abbreviation: PU, perfusion units. 
ing vasoconstriction or whether the vasoconstriction would have occurred in the absence of stimulation.

A methodology to extract and quantify blood flow responses to stimuli from complex signals like laser Doppler flowmetry would allow us to determine individual subject sensitivity to the stimulus and measure the effect of various therapeutic interventions. To maximize the amount of information that can be derived from the physiological responses to pain, it is important, from an information theory perspective, to apply a stimulus that contains a broad spectrum of dynamic patterns. In this paper, we introduce a novel technique, which employs rank-based cross-correlation, to detect the similarity and the response latency between a pseudo-random pattern of pain stimuli and the corresponding physiological response. To the best of our knowledge, this is the first study to apply cross-correlation analysis between the time series of randomized pain pulses and physiological measurements to objectively assess pain responses in humans.

\section{Materials and methods}

\section{Pain protocol and data acquisition}

\section{Subjects and study condition}

The Institutional Review Board at Children's Hospital Los Angeles, California approved the study. For demonstration of the analyses presented in this paper, we analyzed 24 healthy African-American subjects $(30 \pm 12$ years old, 15 females) from a population of subjects recruited as a part of an investigation of ANS response to painful stimuli in SCD. All participants signed informed consent prior to participating in the study. Subjects had to be greater than 13 years of age, not have any serious medical conditions, and be able to sign consent or assent. The total duration of the experiment was approximately 40 minutes after the initial 30 minutes of familiarizing the subject with the experimental protocol and placing sensors. Studies began in the morning in a quiet, dimmed room. The room temperature was set to $25^{\circ} \mathrm{C}$. $^{2}$

\section{Instruments and baseline recording}

As depicted in Figure 2, the subject sat comfortably in a reclining armchair and was asked to stay awake for the entire study period. Measurements of microvascular blood flow were made on the dorsum of left and right index fingers just proximal to the nail bed using laser Doppler flowmetry (LDF; Perimed PeriFlux 5,000 with probe 456 thermostatic small angled probe). The LDF measures the average speed of red blood cell (RBC) particles and their concentration in the microvasculature $1 \mathrm{~mm}$ under the skin. Its principle of operation is based on a Doppler shift effect from light scattered off of red cells moving in an approximately $1 \mathrm{~mm}$ cube, $1 \mathrm{~mm}$ under the skin. This signal cannot be calibrated in absolute units, but changes in the signal have been shown to be directly related to microvascular flow. ${ }^{6,7}$ The product of RBC speed and concentration is proportional to the blood flow, which is represented in perfusion units and is a relative measurement unit. $^{8}$ The sampling rate of the device was set to $32 \mathrm{~Hz}$. The laser probe was placed on the dorsum of the index finger of the hand contralateral to the arm with the pain stimulus. To deliver the thermal stimuli, we placed a neurosensory analyzer thermode (Medoc, TSA-II with a standard $30 \times 30 \mathrm{~mm}$ thermode) on the ventral aspect of the right forearm and programmed it to deliver heat pulses as described in Figure 3. The thermode temperature was recorded along with the physiological responses to permit a precise measurement of time between the heat pulse and the vascular response. We analyzed the microvascular blood flow response measured on the left index finger, contralateral to the arm, where we administered the pain stimulus. The stimulation and response signals were recorded simultaneously using a Biopac MP150 acquisition system. After sensor placements and initial instructions, the protocol began with a 5-minute period of quiet baseline recording.

\section{Heat pain calibration}

After the baseline recording, we calibrated the subjects' pain perceptions to the magnitude of temperatures by applying two sets of three heat ramps rising at $1.5^{\circ} \mathrm{C}$ per second (Task-A in Figure 2). The temperature that was perceived as painful (pain threshold) and intolerably painful (pain tolerance) were determined as follows: during the first set of three heat ramps, the study operator immediately turned off the temperature ramp when the subject reported the minimum sensation of pain. During the second set of three heat ramps, the study operator did the same when the subject asked that the heat be turned off because of the maximum sensation of pain perceived. We defined the pain threshold as the average temperature at which the subject reported first feeling pain, and the pain tolerance as the average of the three temperatures that the subject reported feeling maximum tolerable pain. Subsequent pain stimuli were administered as a series of pulses between the threshold and tolerance temperatures. These sequences are referred to as "pain tasks" (Task-B in Figure 2). ${ }^{2}$

\section{Heat pain stimulation}

The pain task consisted of a series of heat pulses with pseudorandomly selected temperature amplitudes and inter-pulse intervals (Task-B in Figures 2 and 3). The randomness within 


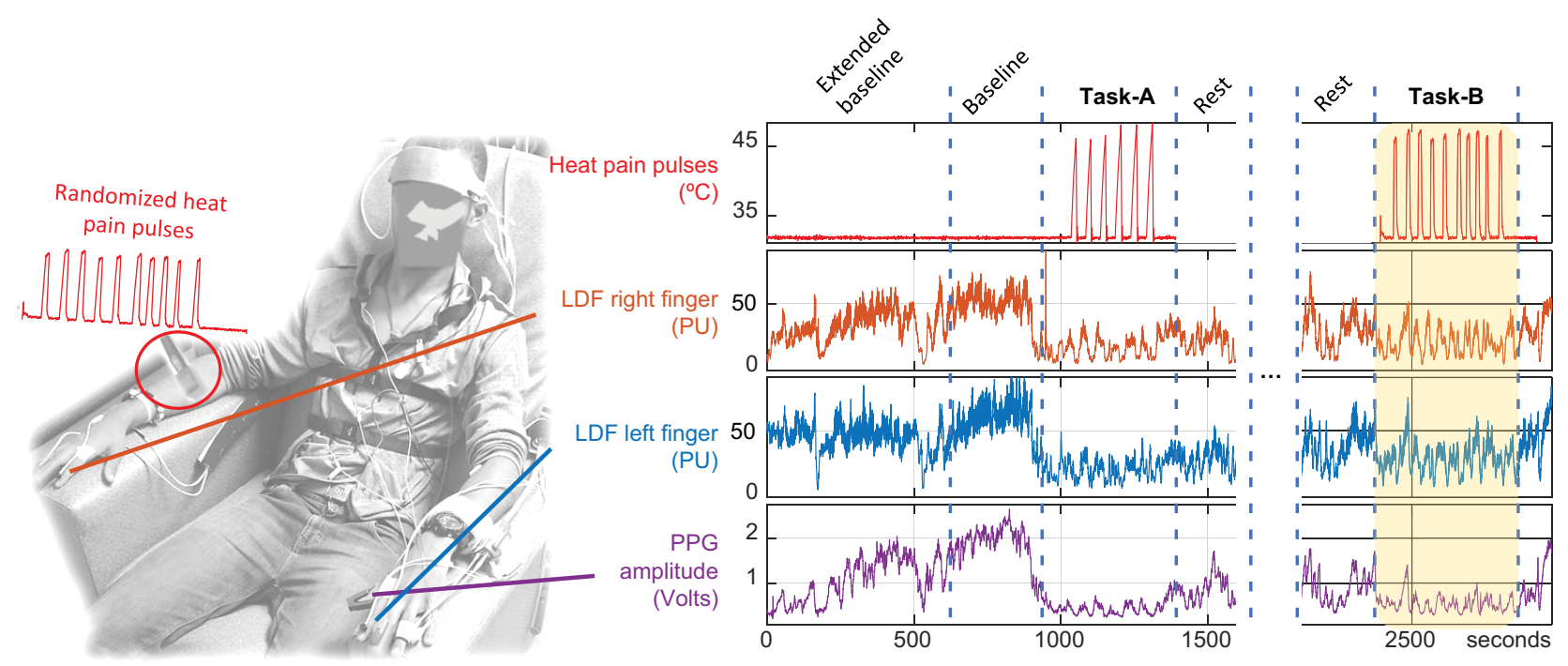

Figure 2 Study setup and measured signals. A subject receiving heat pulses, while finger microvascular blood flow responses are simultaneously recorded.

Notes: Note the three lower height pain threshold pulses followed by the three higher temperature pain tolerance pulses in Task-A. LDF and PPG showed regional vasoconstriction in the finger. Blood flow patterns observed between the left and right fingers are very similar, suggesting that non-pain influences that drive the fluctuations also are central, not so much local. The baseline period used in this study includes both the "extended baseline" and "baseline" periods.

Abbreviations: LDF, laser Doppler flowmetry; PPG, photoplethysmograph; PU, perfusion units.

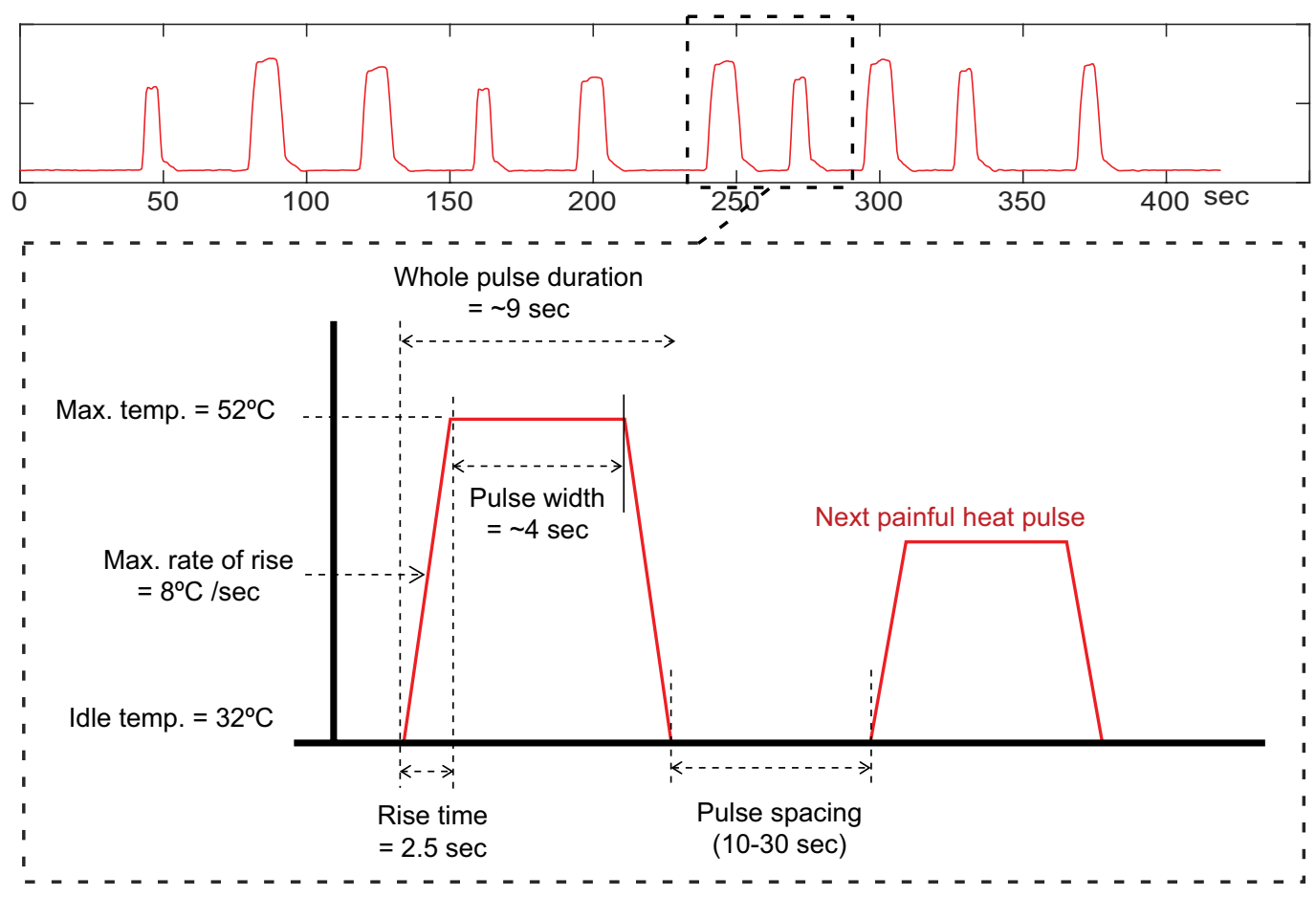

Figure 3 Heat pulse train for Task-B had randomized pulse period and magnitude.

Notes: One rising edge to another rising edge of the painful heat pulse ranged from 19 to 39 seconds (pulse duration + pulse spacing) which corresponded to the peaks at around 0.05 and $0.025 \mathrm{~Hz}$ in Figure 4, respectively. The pulse train also had a slow ( 200 seconds) oscillation in signal magnitude which corresponded to a peak at $0.005 \mathrm{~Hz}$. Note that the randomization sequence was fixed such that every subject had exactly the same pain pattern but with individualized temperature range (pulse period=20.7 \pm 6.5 seconds; heat pulse $=50 \% \pm 40 \%$ pain-range; uniform distribution). The top panel shows one example of the pulse train. The actual pulse duration was often longer than 9 seconds due to hardware controller error, resulting in more pronounced peaks at around $0.04 \mathrm{~Hz}$ as shown in Figure 4 . Average difference between the pain threshold and tolerance of all subjects was $4.2( \pm 2.5)^{\circ} \mathrm{C}$ (temperature ranged from $37.2^{\circ} \mathrm{C}$ to $51^{\circ} \mathrm{C}$; see Figure S2).

the pulse train was intended to minimize the chance of overlap between the pain response and the spontaneous oscillations that usually exist in physiological signals. ${ }^{5}$ The stimulus train consisted of 10 random levels of heat pulses within the pain threshold and tolerance temperature range that was deter- mined in Task-A (Figure 3). Each pulse was programmed to have a 9-second duration followed by inter-pulse periods randomized from 10 to 30 seconds, which were set similar in other previous studies that quantified physiological responses to heat pain. ${ }^{9}, 10$ We used a uniform random distribution for 
creating the heat variation and inter-pulse intervals. This resulted in pronounced peaks in the stimulus power spectrum at 0.025 and $0.04 \mathrm{~Hz}$ (bold red line in Figure 4). About one minute prior to the beginning of the randomized pain task, the subject was instructed to sit still, stay awake, and not to interact with the study operator. This task was preceded and followed by a 5 -minute rest period.

\section{Signal pre-processing for smoothing and normalization}

Recorded signals were processed and analyzed using customized scripts programmed using MATLAB (The Mathworks). All recorded signals were downsampled to $2 \mathrm{~Hz}$ using the "Resample" function in MATLAB to avoid using overly sampled time series while preventing signal aliasing. Then, the frequencies outside of $0.003-0.1 \mathrm{~Hz}$ were filtered out of the blood flow signal using a zero-phase finite impulse response low-pass filter followed by moving average removal method (300-second Hanning window) in order to smooth the signal and remove the cardiorespiratory signal, sharp spikes due to abrupt motion, and other physiological trends (eg, slow signal drifts and baseline changes due to anxiety and other factors). ${ }^{5}$ Further processing may be done to the blood flow signal, so that the VR can be compared between subjects (not shown in this work). For example, the blood flow signal can be normalized to the immediate 5-minute resting period before the pain task (ie, Task-B in Figure 2) using, $Y_{\text {normalized }}=\frac{Y_{\text {task }}-\mu \text { baseline }}{\sigma_{\text {baseline }}}$ where $Y$ is the signal of interest, $\mu$ is the mean of , and $\sigma$ is the $\mathrm{SD}$ of $Y$.

For the pain pulse stimuli signal, we applied a median filter (with a 1.5-second window) to smooth out the high-

A

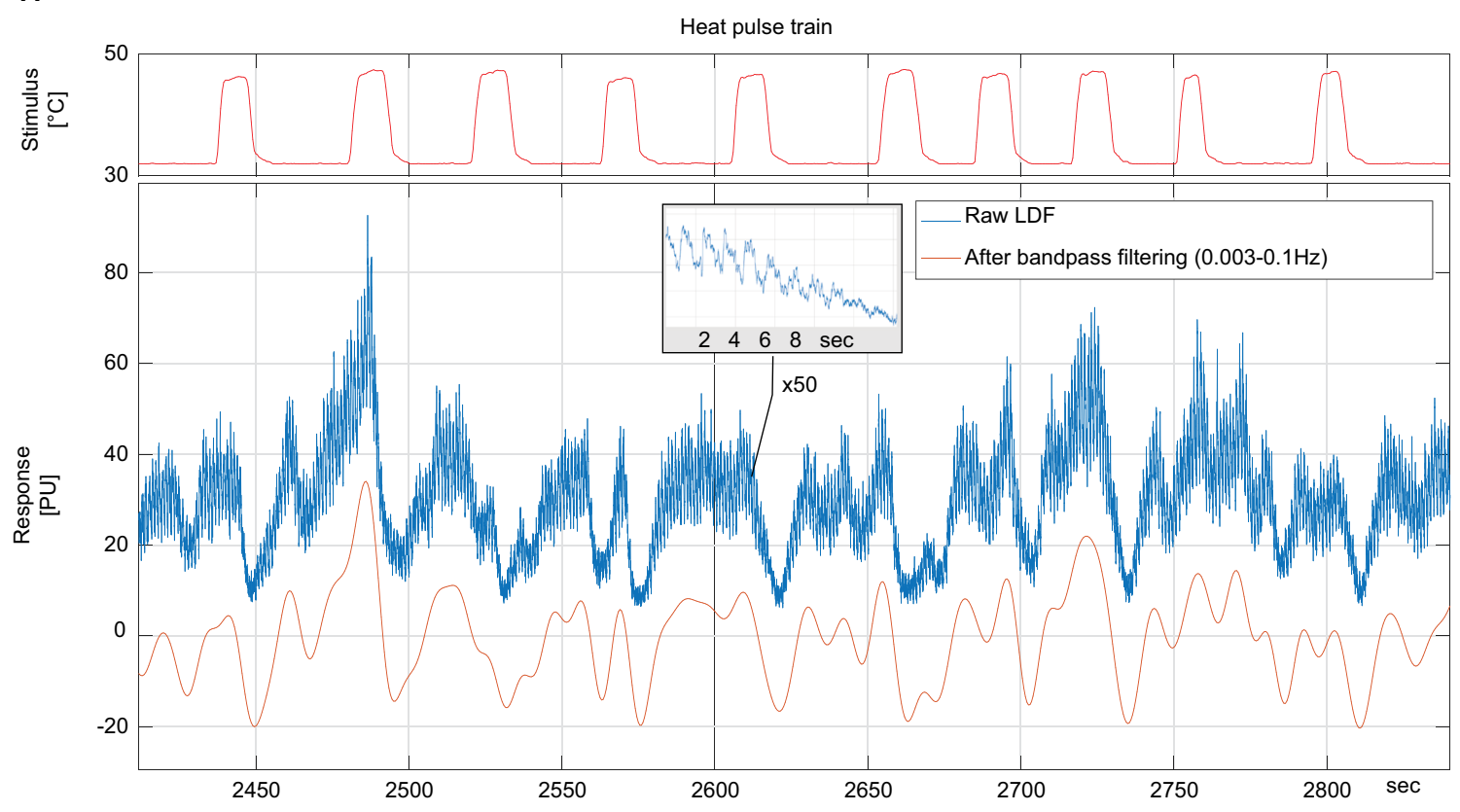

B

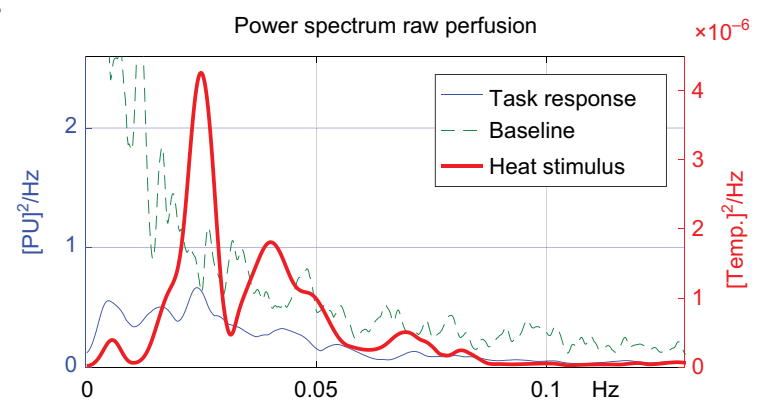

C

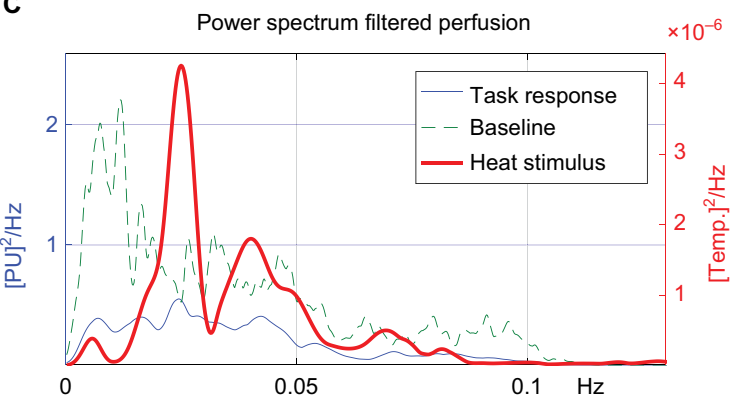

Figure 4 Raw and processed signals.

Notes: (A) The $0.1 \mathrm{~Hz}$ low-pass filtering helped remove pulsatile blood flow and sharp motion artifacts. (B and $\mathbf{C})$ The average frequency responses of the stimulation (bold red), blood flow response (thin blue), and baseline (dashed green) period of all subjects. The power spectral densities indicated clear microvascular blood flow responses in sync with heat stimuli, and their common peaks (especially at $0.025 \mathrm{~Hz}$ ) did not align with that of from spontaneous baseline oscillation. The two plots show power spectral density (PSD) of the blood flow signal before and after applying the bandpass filter. (pass band: $0.003-0.1 \mathrm{~Hz}$ ). 
frequency noise while maintaining the sharp rising and falling edges of the heat pattern (top of Figures 3 and 4). The cutoff frequencies were determined such that the frequencies of interest were maintained. For example, our stimulus pattern covered the frequencies of $0.005-0.050 \mathrm{~Hz}$ range, which included 20-40 seconds pulsing periods, as well as the slow 200 -second oscillation along the envelope of the pain pulses as described in the Figure 3 caption and the power spectral density depicted in bold red in Figure 4.

\section{Computing response indices of correlation, time lag, vasoreactivity, and significance \\ Task-response correlation coefficient $(R)$ and time lag (Tr)}

We computed the cross-correlation between the randomized pain pulses and the pre-processed blood flow signal using Spearman's rank-based correlation method. ${ }^{11,12}$ The pain pattern included the 30 -second local baseline periods before the first pain pulse and after the last pain pulse (see the top of Figure 3). The inclusion of the pre- and post-pain period helped in detecting the responses more accurately at the onset and offset of the whole pain task. The cross-correlation provided the correlation between the pain window and a sliding window of the same number of samples containing the blood flow response. This nonparametric correlation does not assume that blood flow would change linearly with increasing temperature. It is also not overly sensitive to abrupt signal changes due to artifacts and helps minimize the unwanted effects from other noise sources that our band-pass filter could not remove. ${ }^{13}$
The algorithm searched for the values of the correlation coefficient $(\mathrm{R})$ and lagged time $(\mathrm{Tr})$ that corresponded to the most negative correlation between the pain block and the blood flow within a 30 -second response window after onset of the pain task. We focused on the negative correlation because previous findings and our initial observations suggested more robust vasoconstriction responses as the pain temperature increases. ${ }^{14} \mathrm{~A}$ simplified illustration of the cross-correlation is shown in Figure 5. Note that the sign of the heat pulse has been inverted to facilitate comparison of the stimulus and response waveforms in the figure. The arrow indicates the time lag, which is the time it takes to reach the most negative correlation from the stimulus onset within the 30-second window (Figure 5 middle panel, bold red).

\section{Magnitude of vasoreactivity (VR)}

We also estimated the VR by fitting a regression line between the pain pulses and the blood flow change $(y=V R x+B$, where $\mathrm{x}$ represents the time series of pain stimulus, $\mathrm{y}$ is the blood flow response, and $\mathrm{B}$ is the intercept of the regression line). An example of the regressed line is shown in Figure 5B, and the slope of the line represents the VR of the peripheral microvasculature due to pain. As a result, VR represents the change in microvascular blood flow per unit change in temperature ([ $\Delta$ microvascular blood flow $] /[\Delta$ temperature $])$, which is a measure of the sensitivity of the vasoconstriction response to change in temperature. The intercepts $(B)$ were not considered in this paper because the bandpass filter removed the mean and slow changes of the response signal (y); therefore, the true intercept of the response was not retained in our analysis.
A

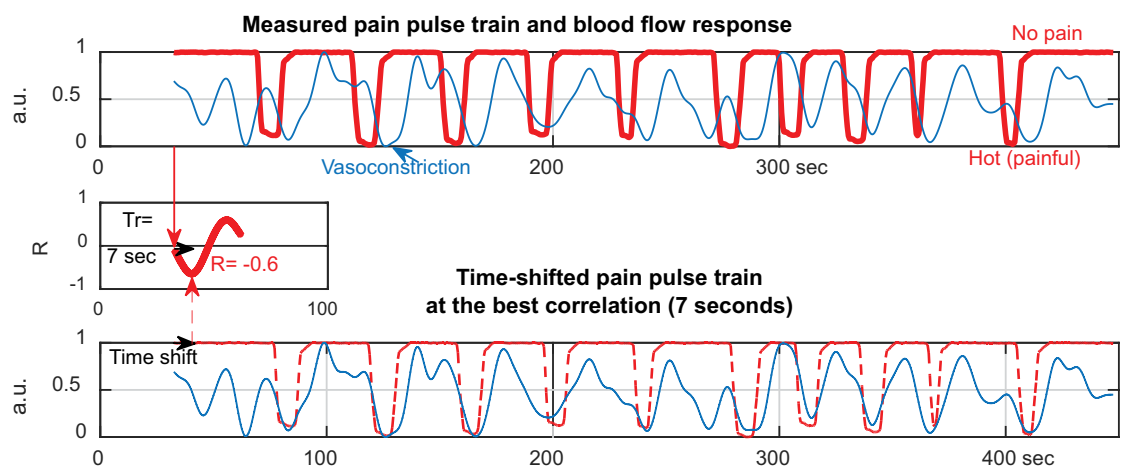

B

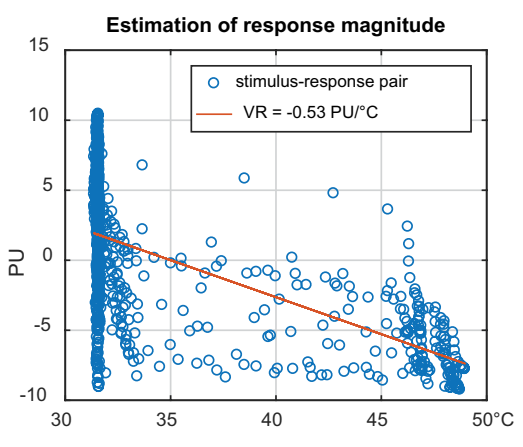

Figure 5 lllustration of a cross-correlation session and corresponding vasoreactivity.

Notes: (A) The correlation between the heat pulses (dashed line) and finger blood flow (blue solid line) after the bandpass refinement shows a clear relationship. In the top panel, the sign of the pain was inverted and signals were normalized to 0-I for an easier comparison. In the middle panel, the small arrow represents the response time delay. The panel (B) shows the regressed line between the stimulus and response. 
Statistical test for match of stimulus pattern in the response data

The correlation coefficient $(\mathrm{R})$ quantifies the direction and the strength of association between the pattern of pain stimuli and the blood flow response. However, it is possible that spurious correlation could arise from naturally occurring fluctuations in blood flow that coincided with the fluctuations in the pain stimuli. To statistically distinguish the pain-induced response from spontaneous fluctuations, we used the bootstrap-based hypothesis testing as illustrated in Figure 6. In detail, we constructed the null distribution by cross-correlating the pain signal with all possible sections of the baseline period (and extended baseline period as defined in Figure 2) and found the strongest correlation within the 30-second lag window as described in the previous section (Figure 5). We collected their correlation coefficients to construct the null distribution. Then, we computed the percentile of the pain task correlation (R) among the null distribution and converted it to the $P$-value (Pr) of a range from 0 to 1. For example, case one in Figure 6 resulted in a more significant $P$-value than that from case 2 , because case one showed more synchronized vasoconstriction response during the pain task compared to the baseline. In contrast, the pain response in case two was not significant because the spontaneous fluctuations during the baseline contained the patterns similar to the stimulus, and hence the cross-correlation at the time of stimulation would not be different than baseline. Examples of computed R and Pr values from the cross-correlation and statistical test are shown in Figure 7. Finally, Pr, Tr, and VR values were used as response indices that can represent an individual's pain response.

\section{Results}

\section{Pain-blood flow correlation and} response time confirm vasoconstriction response

We observed similar LDF measurements obtained from the left (contralateral to painful heat) and right (ipsilateral to painful heat) index fingers, which indicated a central origin that influenced the blood flow, rather than local (Figure 2). Average power spectrum of the stimulus (red) and perfusion response (blue) showed pronounced peaks at 0.025 and 0.04 $\mathrm{Hz}$, as shown in Figure 4. As expected, there was no spectral peak in this range during the baseline observation (green). Using the statistical test described in the previous section, we identified 16 out of 24 subjects in this test dataset who showed statistically significant vasoconstriction responses to heat pain $(\operatorname{Pr}<0.05$, average response time $[\operatorname{Tr}]=6.7 \mathrm{sec}-$ onds). Among seven out of eight nonresponders who also had the LDF measurements from the ipsilateral side of the pain ( $\operatorname{Pr} \geq 0.05)$, five subjects showed insignificant blood flow responses in the ipsilateral side as well; this group includes two subjects who showed consistently unrealistic response delays (Subjects A, B, D, F, and H in Table 1). The rest of the nonresponders (E and $G$ in Table 1) did not show the same level of significance in their ipsilateral side and the contralateral side blood flow responses, which may be due to spatial variation in LDF measurements $(R<0.4)$ that our method could not account for. Similar inconsistency in the Pr values between contralateral flow and ipsilateral flow was observed in 3 out of the 16 responders.

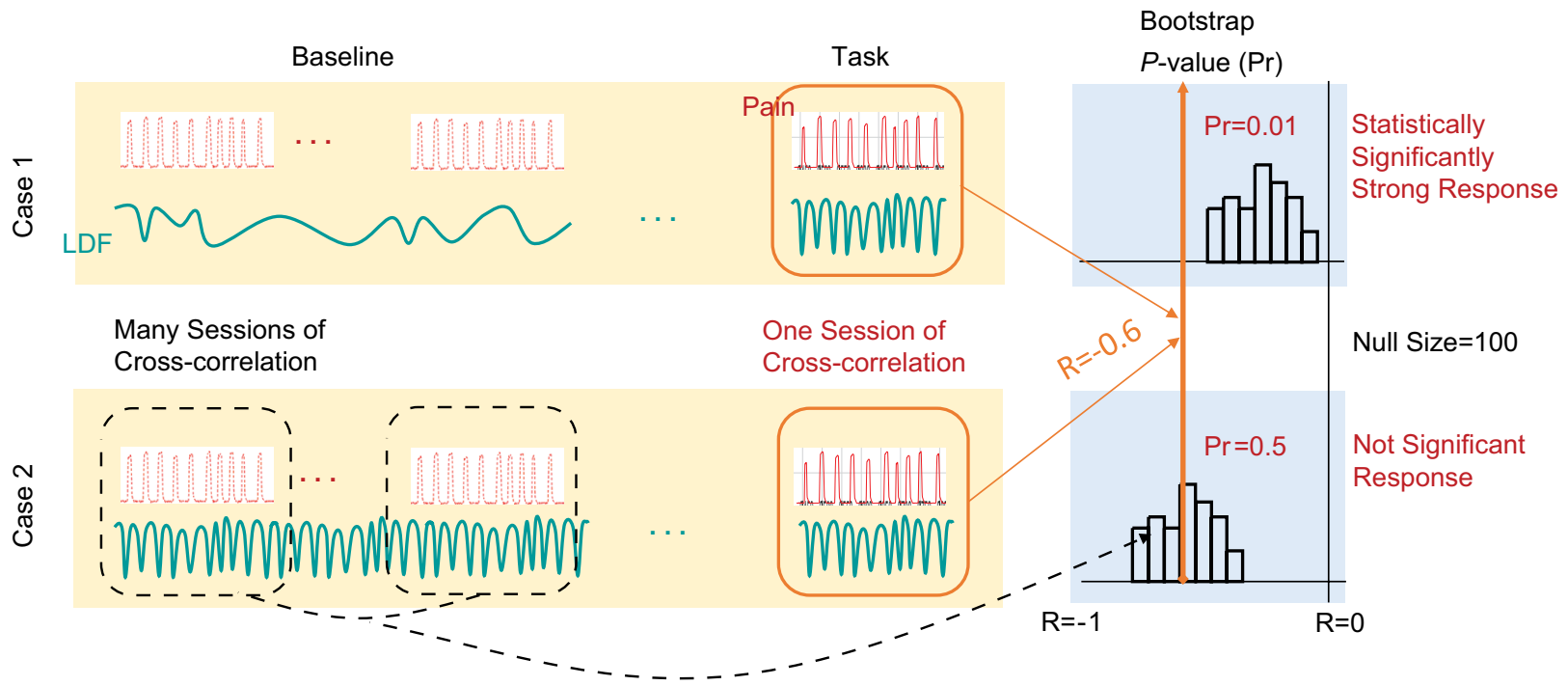

Figure 6 Two hypothetical cases of standardization to a subject's own baseline using a bootstrap test.

Notes: Note that there were no stimuli applied during the baseline recording period, but we used all possible starting points during the baseline period to compute a correlation with the pain pulse. Computed correlation coefficients are collected and a null-response distribution was constructed. Case $I$ and case 2 resulted in different response index (bootstrap $P$-value, or $\mathrm{Pr}$ ) after the test, but originally had the same task-response correlation coefficient (R). 
A

$R=-0.64, \operatorname{Tr}=7.5 \mathrm{~s}, \mathrm{Pr}=0.0005$

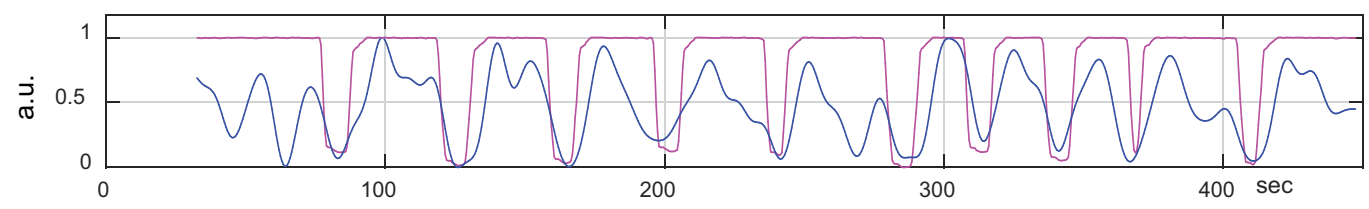

B

$R=-0.11, T r=26.5 s, \operatorname{Pr}=0.3567$
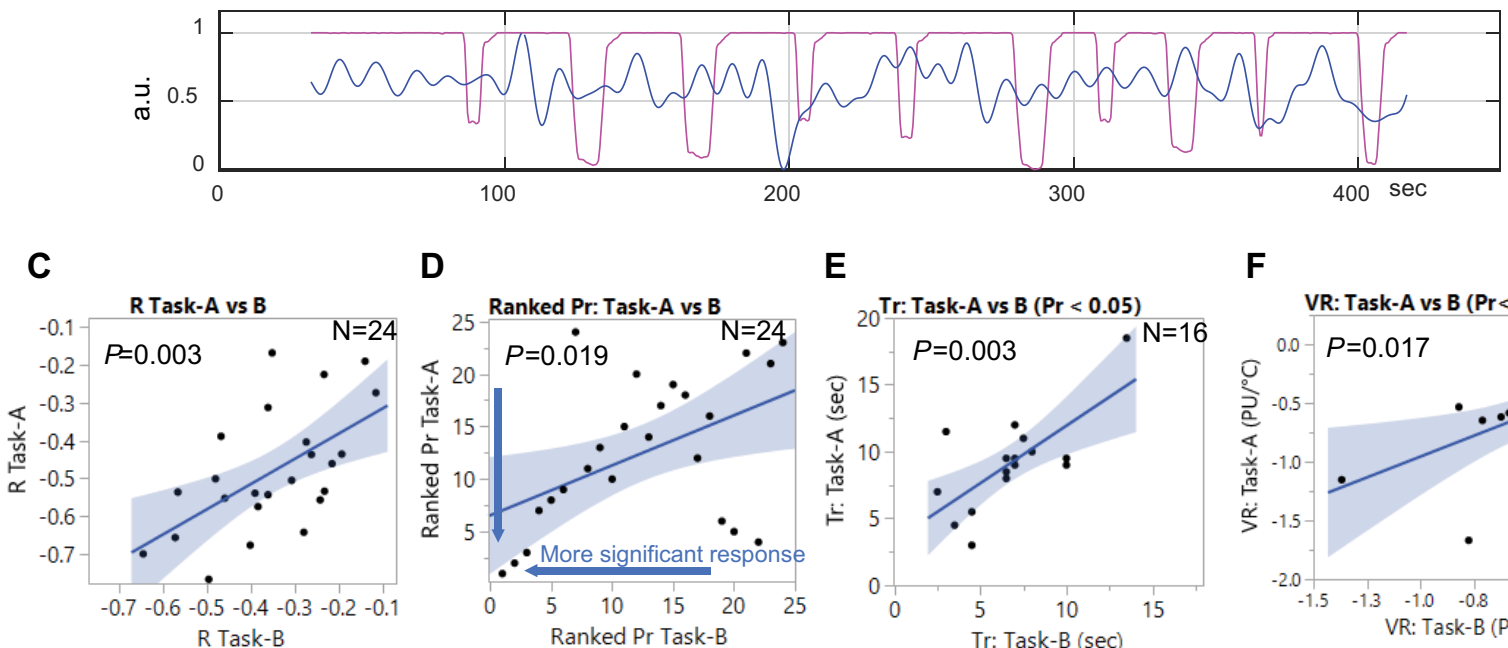

D

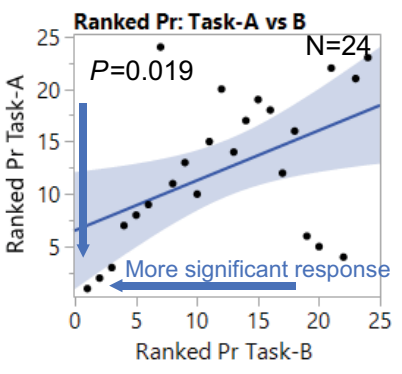

E

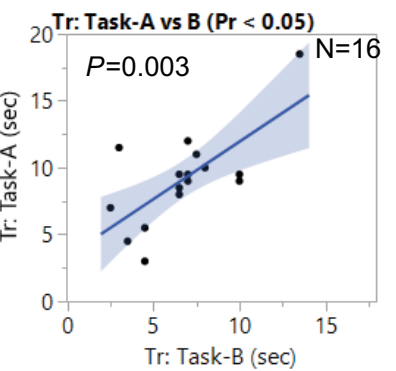

$\mathbf{F}$

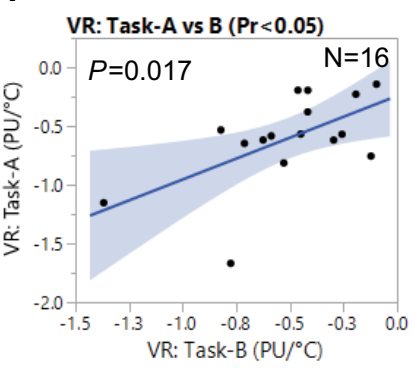

Figure 7 Examples of significant and insignificant correlations between heat pulses and blood flow response, and response comparison between Task-A and Task-B. Top two rows: (A) strong and (B) weak correlations against their own null responses. The response and heat signals are scaled to unity. Heat pulses are inverted and shifted to the time of best correlation with blood flow for easy visualization. Bottom row: comparison of the estimated (C) correlation coefficient, (D) significance of the correlation, (E) time to respond, and (F) vasoreactivity from Task-A and Task-B. Each dot represents a subject showing a similar response across two similar tasks.

Abbreviations: $\mathrm{R}$, correlation coefficient; $\mathrm{Pr}$, bootstrap $\mathrm{P}$-value; $\mathrm{Tr}$, response time.

Representative test results are illustrated in Figure 7, showing an example of (A) strong and (B) weak correlations against their own baseline null responses. For the visual purpose, the heat pulse train in Figure 7 was inverted and normalized to a scale of $0-1$ and was superimposed on the blood flow. You can see the response coincides with the stimulus in the subject with the significant $\operatorname{Pr}$ values. The association of stimulus to response is unclear in the subject (B) where the Pr value is not significant. The graphs at the bottom of Figure 7 show the relation of R, Pr, Tr, and VR for two different pain tasks within the same subject. Each of the R, Pr, Tr, and VR indices shows strong correlation between two different pain challenges in the same subject, which suggests that these derived parameters represent a biological characteristic of the subject. Examples of subjects who showed significant (or insignificant) responses in both Task-B and Task-A are shown in Figure S1.

\section{Discussion}

We have introduced a new method based on cross-correlation analysis for objectively quantifying the microvascular blood flow response to painful heat pulses. Using a stimulation pattern of randomly spaced and sized thermal pulses, we were able to determine the microvascular responses with high temporal resolution. The estimated correlation between the stimulus and response was statistically tested against each subject's own spontaneous fluctuations at the baseline where there was no stimulation. Finally, our method estimated a set of response indices (of the response correlation, response time, vasoreactivity, and response significance) that can be used for characterizing the ANS-mediated microvascular blood flow responses to various pain stimuli and may be useful for understanding conditions such as altered pain perception and ANS dysfunction in disease processes. While we have used the heat pulses to induce pain and measured peripheral microvascular blood flow as the response to the pain stimulation, our technique can be generalized for analyzing other signal modalities, such as the measurements in finger photoplethysmogram (PPG; see Supplementary materials), electrocardiogram, and functional brain imaging.

The quantitative sensory testing (QST) technique finds sensory thresholds to various types of pain stimulation but 
Table I Comparison of microvascular blood flow responses between contralateral and ipsilateral sides of the pain for nonresponders

\begin{tabular}{|c|c|c|c|c|c|c|}
\hline \multirow[t]{2}{*}{$\overline{I D}$} & \multicolumn{2}{|c|}{$\begin{array}{l}\text { Response significance } \\
\text { (P-value, } \mathrm{Pr} \text { ) }\end{array}$} & \multicolumn{2}{|l|}{$\begin{array}{l}\text { Response time } \\
\text { (seconds, Tr) }\end{array}$} & \multirow{2}{*}{$\begin{array}{l}\text { Contralateral } \\
\text { and ipsilateral } \\
\text { flow correlation } \\
\text { coefficient }(R)\end{array}$} & \multirow{2}{*}{$\begin{array}{l}\text { Null } \\
\text { sample } \\
\text { size }\end{array}$} \\
\hline & $\begin{array}{l}\text { Contralateral } \\
\text { finger flow }\end{array}$ & $\begin{array}{l}\text { Ipsilateral } \\
\text { finger flow }\end{array}$ & $\begin{array}{l}\text { Contralateral } \\
\text { finger flow }\end{array}$ & $\begin{array}{l}\text { Ipsilateral } \\
\text { finger flow }\end{array}$ & & \\
\hline $\bar{A}$ & 0.633 & 0.339 & $19.5^{\mathrm{a}}$ & $18^{a}$ & 0.79 & 2,335 \\
\hline B & 0.357 & 0.825 & $26.5^{\mathrm{a}}$ & 13 & 0.5 & 965 \\
\hline$C^{b}$ & 0.236 & - & $29^{a}$ & - & - & I,545 \\
\hline $\mathrm{D}$ & 0.182 & 0.059 & 3.5 & 5 & 0.65 & 1,078 \\
\hline$E$ & 0.143 & $0.00 I^{c}$ & 2 & 3.5 & $0.39^{d}$ & $\mathrm{I}, 443$ \\
\hline$F$ & 0.115 & 0.109 & 7.5 & 8.5 & 0.54 & 1,519 \\
\hline G & 0.090 & $0.00 I^{c}$ & 9 & 6 & $0.38^{d}$ & 751 \\
\hline $\mathrm{H}$ & 0.063 & 0.072 & $28^{a}$ & $27.5^{\mathrm{a}}$ & 0.73 & I,008 \\
\hline
\end{tabular}

Notes: anrealistically long response delay ( $\operatorname{Tr}>15)$; 'subject C's ipsilateral LDF measurement was rejected due to excessive signal noise and artifacts due to instrumentation; 'significant blood flow response; ${ }^{\mathrm{d}} \mathrm{R}<0.4$.

Abbreviations: LDF, laser Doppler flowmetry; Pr, bootstrap $P$-value; $\mathrm{Tr}$, response time.

relies on subjective reporting that depends on the subject's ability to verbalize the pain intensity. ${ }^{15}$ While central nervous system influences can modulate microvascular blood flow and contribute to variability, the ability to directly measure the blood flow in response to pain provides an objectively quantifiable endpoint that is physiologically relevant to vaso-occlusion in sickle cell patients. Signal averaging of repetitions of responses to the same magnitude and duration of pulses can also be used to detect responses in noisy signals. ${ }^{10}$ This technique helps increase the signal-to-noise ratio and identify more reliable shape of the pain response in time; however, the practical need to employ short study duration limits the variety of stimulus patterns that can be applied and consequently the breadth of responses that can be detected. ${ }^{16}$ There are also coherence-based techniques that quantify and compare the phases and/or amplitudes of the frequency components in the stimulus and response. ${ }^{17}$ However, interpreting results in frequency domain is not as intuitive as comparing the stimulus and response signals in time domain. There are previous reports that quantified mathematical features such as response amplitude, stationarity, entropy, linearity, and variability changes against the baseline activity. ${ }^{9,18}$ Yet, these methods still require multiple repetitions of the stimulus to obtain statistical significance of the quantified response.

We have identified the responders and nonresponders to pain by using the response significance $(\mathrm{Pr})$ as an index. Assuming the pain response was from a central origin, we anticipated that our Pr indices obtained from both the contralateral and ipsilateral sides of the pain would agree to each other, and this would further validate our method. Yet, there were few subjects who showed inconsistency as shown in Table 1 . Nonetheless, about $80 \%$ of the subjects showed consistent responses between two simultaneously sampled regions of the body quantified using our method, and we also found similar consistency when comparing the results obtained from two different tasks (ie, Task-A and Task-B; see Figure S1). Moreover, we analyzed the responses of PPG amplitude and confirmed that the methods described here work equally well and yield similar results, showing that the central origin was an important factor, and that the proposed method could also be applied to the PPG signal (see Table S1).

Our correlation-based approach required a longer baseline period than the test stimulus pattern itself. For example, our pain task duration was 7 minutes, and cross-correlating that pattern with 7 minutes of the baseline recording only gave one R-value. In order to have a reasonable $P$-value resolution, for example, 0.025 , one would need $40 \mathrm{R}$-values as null samples. However, it is not realistic to record 40 unique and independent snippets of 7-minute responses in one study (that is, $\sim 5$ hours of baseline recording itself). To overcome this limitation, we assumed we were obtaining another cycle of unique oscillatory response in every 10 seconds of blood flow. ${ }^{3,19}$ Based on this effective unit of time, we would need another $40 \times 10$ seconds $=400$ seconds $=\sim 7$ minutes of baseline data in addition to the length of the task stimulation to obtain bootstrap $P$-value with reasonable robustness and resolution. For this reason, we constructed the null distribution by combining the 5 -minute silent baseline recording and 10-15 minutes of the extended baseline recording periods (Figure 2). This resulted in an average of 1,208 \pm 492 samples 
in $2 \mathrm{~Hz}$ or about $60 \pm 25$ effective samples $(P$-value resolution of $\sim 0.017$ ) in the null distribution for each subject.

The inter-pulse periods of 10 30 seconds can be a concern as the finger blood flow may not have recovered to its original baseline flow before the next pain stimuli and can create a stacking effect on the subsequent blood flow responses to pain. Thus, the inter-pulse timing must be optimized based on the particular stimulus and response being studied. Our quantification method is still robust if the stacking effect is small. Previously, we have shown that the peripheral vasoconstriction responses to heat pain occurred rapidly and were detectable using the pulses described here. ${ }^{3}$

Finally, although regular tidal breathing exerts only a small influence on microvascular blood flow, excessively irregular breathing patterns can significantly confound the effects of thermal pain on peripheral blood flow. We previously found that sighs can provoke significant transient episodes of peripheral vasoconstriction via a sympathetically mediated reflex. ${ }^{4}$ This confounding influence is only problematic when it occurs at the time of the pain stimulus because the sighvasoconstriction response can eclipse the true pain response. One solution is to apply adaptive filtering techniques to filter out the respiration and to determine the partial correlation after controlling for the respiration with a certain delay. ${ }^{20}$ Nevertheless, we applied several randomized pain pulses in one pain task to reduce the chance of the confounding events. The fact that we observed strong similarity in response indices from the similar pain pulse sequences (Task-A and Task-B) in the same subject suggests that the interference from the respiration did not significantly affect the analysis.

\section{Conclusion}

We have introduced a novel method for objective quantification of the regional microvascular blood flow response to painful heat pulses. Unlike previous studies, we designed a painful pulse train that created many short-term heat sensations in randomized magnitude and periods. We computed the response correlation, response time, and vasoreactivity between the pain pulse train and the blood flow change using laser Doppler flowmetry, and obtained the $P$-value of the response using a bootstrapping method. These computed values serve as indices of pain response, and we believe our pain indices can provide additional information compared with other conventional pain response tests and questionnaires. While we have used heat to induce pain and blood flow responses, our technique can be adapted to different modalities of stimulus and response measurements for extracting other psychological determinants of diseases related to pain or vascular reactivity.

\section{Acknowledgments}

The authors would like to thank Dr Saranya Veluswamy for her review of the manuscript, and Wanwara Thuptimdang, Leonardo Nava, Dr Herbert J. Meiselman, and Dr Henry J. Forman for helpful discussions and their feedback throughout this research. This work was supported by National Institutes of Health grants U01-HL117718 (TCD, MCKK, JCW, LKZ) and P41-EB001978 (MCKK).

\section{Author contributions}

JS ran the pain study, analyzed and interpreted the data, and wrote the paper; JS and PC developed the algorithm for signal processing and cross-correlation analysis; PC, MK, and PS ran the pain study, contributed in interpreting the data, and drafting the article critically; RS designed bootstrapping statistical analysis; RMK assisted in design of physiologic measurements; RMK, JCW, and JD contributed in designing the pain study and interpreted the data; LKZ provided substantial contribution in designing the pain study and interpreting the data; TDC and MCKK have designed the study, came up with methodology based on cross-correlation of stimulus and response, and revised the manuscript critically for interpretation of the proposed method and data. All authors contributed toward data analysis, drafting and critically revising the paper and agree to be accountable for all aspects of the work.

\section{Disclosure}

The authors report no conflicts of interest in this work.

\section{References}

1. Bhatt RR, Martin SR, Evans S, et al. The effect of hypnosis on pain and peripheral blood flow in sickle-cell disease: a pilot study. J Pain Res. 2017;10:1635-1644.

2. Khaleel M, Puliyel M, Shah P, et al. Individuals with sickle cell disease have a significantly greater vasoconstriction response to thermal pain than controls and have significant vasoconstriction in response to anticipation of pain. Am J Hematol. 2017;92(11):1137-1145.

3. Chalacheva P, Khaleel M, Sunwoo J, et al. Biophysical markers of the peripheral vasoconstriction response to pain in sickle cell disease. PLoS One. 2017;12(5): 0178353.

4. Sangkatumvong S, Khoo MC, Kato R, et al. Peripheral vasoconstriction and abnormal parasympathetic response to sighs and transient hypoxia in sickle cell disease. Am J Respir Crit Care Med. 2011;184(4):474-481.

5. Stefanovska A. Coupled Oscillatros: Complex but not complicated cardiovascular and brain interactions. IEEE Engineering in Medicine and Biology Magazine. 2006;26(6):25-29.

6. Fredriksson I, Fors C, Johansson J. Laser Doppler flowmetry - a theoretical framework. Department of Biomedical Engineering. Linköping University. 2007. Available from: www.imt.liu.se/bit/ldf/ldfmain.html. Accessed July 3, 2017.

7. Fredriksson I, Larsson M, Stromberg T. Laser Doppler flowmetry. In: Leahy MJ, editor. Microcirculation Imaging. John Wiley \& Sons, Incorporated; 2012:67-86. Available from: http://site.ebrary.com/lib/ uscisd/Doc?id=10565152\&ppg=88. Accessed September 3, 2018. 
8. Perimed AB. Laser Doppler Monitoring. Available from: http://www. perimed-instruments.com/laser-doppler-monitoring. Accessed July 3, 2017.

9. Walter S, Gruss S, Limbrecht-Ecklundt K, et al. Automatic pain quantification using autonomic parameters. Psychol Neurosci. 2014;7(3):363-380

10. Kong J, Loggia ML, Zyloney C, Tu P, Laviolette P, Gollub RL. Exploring the brain in pain: activations, deactivations and their relation. Pain. 2010;148(2):257-267.

11. Minakuchi E, Ohnishi E, Ohnishi J, et al. Evaluation of mental stress by physiological indices derived from finger plethysmography. J Physiol Anthropol. 2013;32:(1):1.

12. Conover WJ, Iman RL. Rank Transformation as a bridge between parametric and nonparametric statistics. Am Stat. 1981;35(3):124-129. Available from: http://people.umass.edu/bioep740/topics/amstat-1985iman.pdf.

13. Gibbons JD, Chakraborti S. Nonparametric Statistical Inference. New York, NY: Marcel Dekker Inc.; 2003. Available from: https://www. scribd.com/document/331131074/NonParametric-Statistical-InferenceGibbons-J-pdf. Accessed November 30, 2017.

14. Hamunen K, Kontinen V, Hakala E, Talke P, Paloheimo M, Kalso E. Effect of pain on autonomic nervous system indices derived from photoplethysmography in healthy volunteers. Br J Anaesth. 2012;108(5): 838-844.
15. Shy M, Frohman E, So Y, et al. Quantitative sensory testing: Report of the Therapeutics and Technology Assessment Subcommittee of the American Academy of Neurology. Neurology. 2003;60(6): 898-904.

16. Cui X, Bray S, Bryant DM, Glover GH, Reiss AL. A quantitative comparison of NIRS and fMRI across multiple cognitive tasks. Neuroimage. 2011;54(4):2808-2821.

17. Mück-Weymann ME, Albrecht HP, Hager D, Hiller D, Hornstein OP, Bauer RD. Respiratory-dependent laser-Doppler flux motion in different skin areas and its meaning to autonomic nervous control of the vessels of the skin. Microvasc Res. 1996;52(1):69-78.

18. Ben-Israel N, Kliger M, Zuckerman G, Katz Y, Edry R, Mark Kliger B, Galit Zuckerman B. Monitoring the nociception level: a multi-parameter approach. J Clin Monit Comput. 2013;27(6):659-668.

19. Bernardi L, Hayoz D, Wenzel R, et al. Synchronous and baroceptorsensitive oscillations in skin microcirculation: evidence for central autonomic control. Available from: http://ajpheart.physiology.org/ content/ajpheart/273/4/H1867.full.pdf. Accessed August 11, 2017.

20. Ljung L. System Identification: Theory for the User. Englewood Cliffs, NJ: Prentice-Hall; 1987.

21. Allen J. Photoplethysmography and its application in clinical physiological measurement. Physiol Meas. 2007;28(3):R1-R39. 


\section{Supplementary materials}

Photoplethysmography (PPG) compared to laser Doppler flowmetry (LDF)

Photoplethysmography (PPG) is another optical technique that uses two wavelengths of light which are differently absorbed by the oxygenated- and deoxygenated-hemoglobin in the vasculature. ${ }^{21}$ Thus, the amount of change of the light intensity due to the body tissue is proportional to the vasodilation or vasoconstriction of the vasculature in the tissue. When applied on a finger, the PPG device measures the changes in the blood volume across the whole fingertip, while laser Doppler flowmetry (LDF) only measures from the small region of subcutaneous skin. The pulsatile part of the PPG signal reflects the changes in the blood vessel diameter from one heartbeat to another, and the height of this PPG pulses is usually correlated with vasodilation or vasoconstriction. ${ }^{2,21}$

\section{LDF- vs PPG-based response indices}

The height of the PPG pulses was extracted (photoplethysmograph amplitude [PPGamp]), followed by the same preprocessing steps for the LDF signal. ${ }^{2,3,21}$ We observed that the LDF and PPGamp signals were usually correlated well (not shown). We also computed R, Tr, and Pr indices to pain, using the PPGamp signal, which showed no statistical differences compared with the indices obtained using the LDF (Table S1). This suggested the PPGamp as an alternative measurement of the peripheral vascular response to pain, as well as that the LDF is capable of detecting microvascular blood flow changes elicited by pain.

\section{Subjective pain-reporting temperatures}

The result obtained from Task-A showed the distribution of pain threshold and tolerance temperatures as well as their ranges (Figure S2).

Table SI LDF vs PPGamp comparison of computed pain response indices

\begin{tabular}{lllll}
\hline & $\begin{array}{l}\text { Response } \\
\text { index to } \\
\text { random pain }\end{array}$ & $\begin{array}{l}\text { Median (IQR) } \\
\text { Pain response index } \\
\text { computed using LDF }\end{array}$ & $\begin{array}{l}\text { Pain response index } \\
\text { computed using PPGamp }\end{array}$ & $\begin{array}{l}\text { Wilcoxon } \\
\text { signed rank } \\
\text { test (P-value) }\end{array}$ \\
\hline Pain task & $\mathrm{R}$ & $-0.35(-0.46$ to -0.23$)$ & $-0.33(-0.43$ to -0.28$)$ & 0.559 \\
$(\mathrm{~N}=24)$ & $\operatorname{Tr}(\mathrm{sec})$ & $7(4.5-10)$ & $7.25(5.6-9.3)$ & 0.749 \\
& $\operatorname{Pr}$ & 0.0010 & 0.0010 & 0.815 \\
& & $(0.0009-0.0800)$ & $(0.0006-0.0608)$ & \\
\hline
\end{tabular}

Notes: Twenty-four healthy subjects were analyzed.

Abbreviations: PPGamp, photoplethysmograph amplitude; LDF, laser Doppler flowmetry; R, correlation coefficient; $\mathrm{Tr}$, response time; Pr, response significance P-value. 

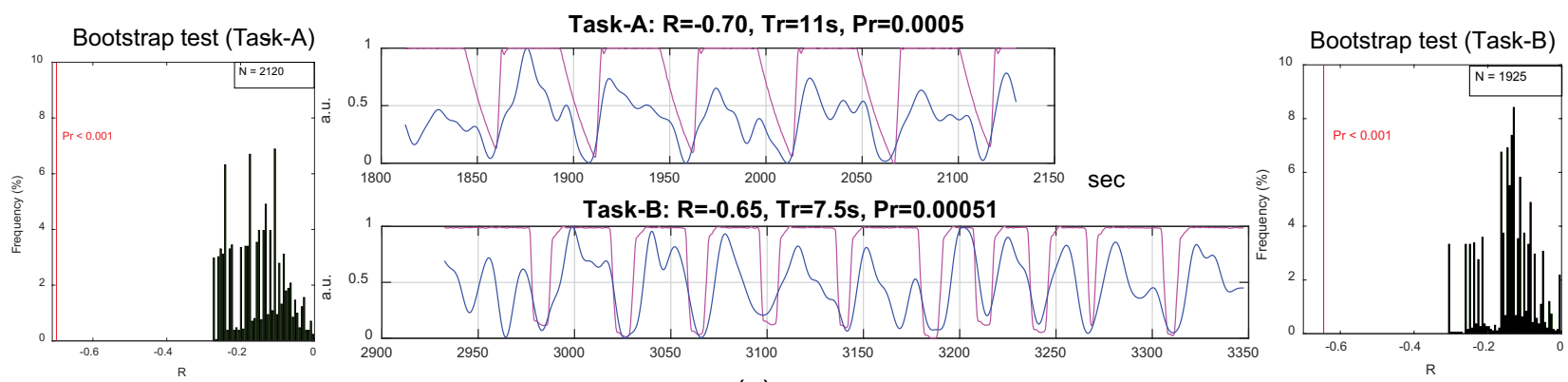

(a)
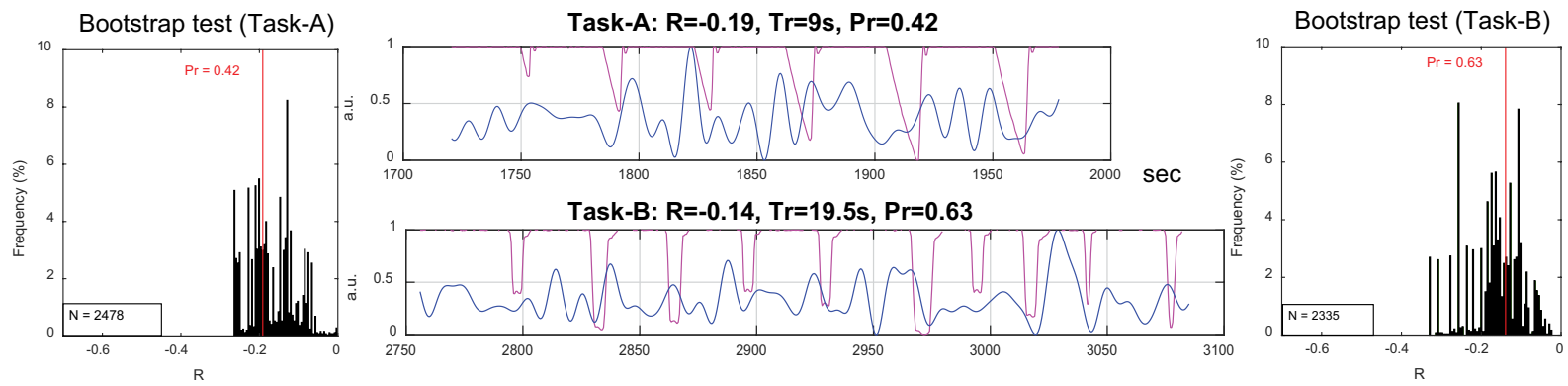

(b)

Figure SI Task-A vs Task-B comparison of the two representative subjects. Null distributions of Task-A and Task-B are on the left and the right columns, respectively. A total of 19/24 ( $80 \%)$ of the subjects showed a consistent identification as either responders or nonresponders across Task-A and Task-B (responder criterion: Pr $<0.05,16$ responders and 3 nonresponders). Case (A) shows one of the consistent responders and (B) shows one of the consistent nonresponders across the two tasks. Four subjects showed significant response only during Task-A and other one subject showed significant response only during Task-B. Variances in the response significance, response times, and correlation coefficients may be due to different stimulation types and interactions during the task.

Abbreviations: $\mathrm{R}$, correlation coefficient; $\mathrm{Tr}$, response time; $\mathrm{Pr}$, response significance $P$-value.

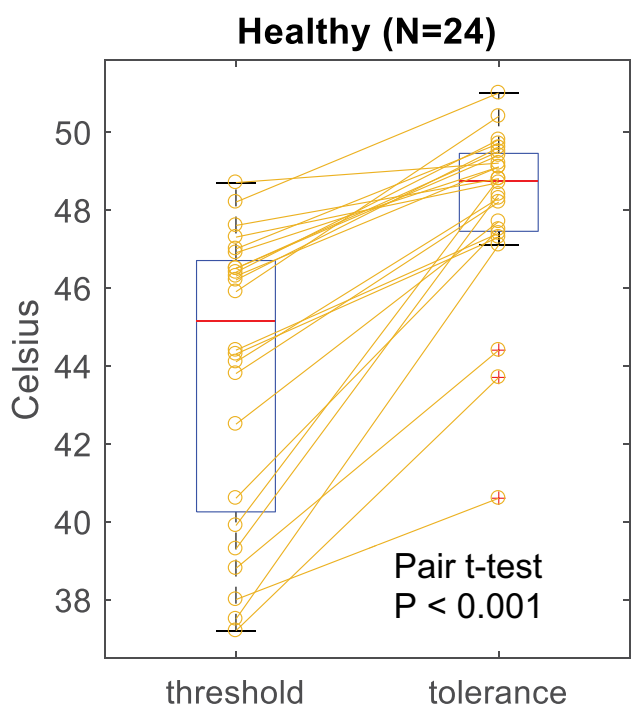

\begin{tabular}{|l|l|}
\hline & Healthy $(\mathrm{N}=24)$ \\
\hline Pain threshold $\left({ }^{\circ} \mathrm{C}\right)$ & $43.9 \pm 3.7$ \\
\hline Pain tolerance $\left({ }^{\circ} \mathrm{C}\right)$ & $48.1 \pm 2.3$ \\
\hline Pain range $\left({ }^{\circ} \mathrm{C}\right)$ & $4.2 \pm 2.5$ \\
\hline Age $($ years $)$ & $30 \pm 12$ \\
\hline Female/Male & $15 / 9$ \\
\hline
\end{tabular}

Figure S2 Heat temperature and range of reported pain threshold and pain tolerance.

Journal of Pain Research

\section{Publish your work in this journal}

The Journal of Pain Research is an international, peer reviewed, open access, online journal that welcomes laboratory and clinical findings in the fields of pain research and the prevention and management of pain. Original research, reviews, symposium reports, hypothesis formation and commentaries are all considered for publication.
Dovepress

The manuscript management system is completely online and includes a very quick and fair peer-review system, which is all easy to use. Visit http://www.dovepress.com/testimonials.php to read real quotes from published authors. 